\title{
The ABCD of Obesity: An EASO Position Statement on a Diagnostic Term with Clinical and Scientific Implications
}

\author{
Gema Frühbeck ${ }^{a, b}$ Luca Busetto $^{a, c}$ Dror Dicker $^{\text {a, d }}$ Volkan Yumuk $^{\text {a, e }}$ \\ Gijs H. Goossens ${ }^{a}{ }^{f} \quad$ Johannes Hebebrand ${ }^{a}, g$ Jason G.C. Halford ${ }^{a, h}$ \\ Nathalie J. Farpour-Lambert ${ }^{a}$ i $\quad$ Ellen E. Blaak ${ }^{a}{ }^{f}$ Euan Woodward ${ }^{a, j}$ \\ Hermann Toplak ${ }^{a, k}$ \\ ${ }^{a}$ European Association for the Study of Obesity (EASO), Teddington, UK ; ${ }^{b}$ Department \\ of Endocrinology and Nutrition, Clínica Universidad de Navarra, University of Navarra, \\ IdiSNA, CIBEROBN, Spanish Health Institute Carlos III, Pamplona, Spain; ' Department of \\ Medicine, University of Padova, Padova, Italy; ${ }^{d}$ Department of Internal Medicine D and \\ Obesity Clinic, Hasharon Hospital, Rabin Medical Center, Petah Tikva, Sackler School of \\ Medicine, Tel Aviv University, Tel Aviv, Israel; ${ }^{e}$ Division of Endocrinology, Metabolism and \\ Diabetes, Department of Medicine, Istanbul University Cerrahpasa Medical Faculty, Istanbul,

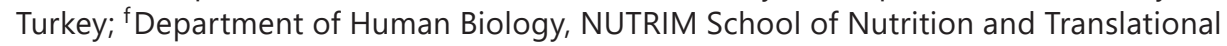 \\ Research in Metabolism, Maastricht University Medical Centre, Maastricht, The Netherlands; \\ 9Department of Child and Adolescent Psychiatry, Psychosomatics and Psychotherapy,

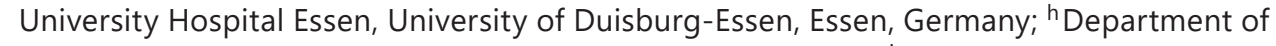 \\ Psychological Sciences, University of Liverpool, Liverpool, UK; ' Obesity Prevention and Care \\ Program Contrepoids, Service of Therapeutic Education for Chronic Diseases, Department \\ of Community Health, Primary Care and Emergency, Geneva University Hospitals and \\ University of Geneva, Geneva, Switzerland; ${ }^{\mathrm{E} E A S O}$ Secretariat, Middlesex, UK; ${ }^{\mathrm{k}}$ Department \\ of Internal Medicine, Medical University of Graz, Graz, Austria
}

\section{Keywords}

Adiposity · Dysfunctional adipose tissue $\cdot$ Noncommunicable diseases $\cdot$ Comorbidities

\begin{abstract}
Obesity is a frequent, serious, complex, relapsing, and chronic disease process that represents a major public health problem. The coining of obesity as an adiposity-based chronic disease $(A B C D)$ is of particular relevance being in line with EASO's proposal to improve the International Classification of Diseases ICD-11 diagnostic criteria for obesity based on three dimensions, namely etiology, degree of adiposity, and health risks. The body mass index as a unique measurement of obesity does not reflect the whole complexity of the disease. Obesity complications are mainly determined by 2 pathological processes, i.e., physical forces (fat mass disease) as well as endocrine and immune responses (sick fat disease), which are embedded in a cultural and physical context leading to a specific $A B C D$ stage.


Frühbeck et al.: The ABCD of Obesity

\section{The Persistent Problem of Obesity}

In spite of significant investments into researching obesity, the battle of halting the epidemic in the past decades has been lost [1-4]. In order to curb this situation, the obesity epidemic should be strategically tackled at the global level.

Obesity is a chronic disease producing an increasingly heavy burden on citizens, health care systems, workforce productivity, cities, and society at large. It should therefore be considered as a top priority, with increased commitments for concerted, coordinated, and specific actions. During the last decade, the European Association for the Study of Obesity (EASO) has been extraordinarily active in promoting actions through collaboration in advocacy, communication, education, and research to reduce the global burden associated with unhealthy body weight. This mission has been accomplished, among others, by highlighting the rising public health, clinical, scientific, economical, and societal challenges caused by obesity [5], as well as by reviewing the current International Classification of Diseases ICD-10 and revising the ICD-11 Beta Draft (July 27, 2018) for the definition of overweight and obesity to propose a substantial revision [6]. Further efforts to improve the assessment and management of children and adults with obesity in a clinically meaningful way include identification of paradigm shifts, calls for action, clinical guidelines, position statements, and education of health care workers [7-12]. Similar efforts have been undertaken by our colleagues in North America [13-15]. Among their diverse activities, the coining of obesity as an adiposity-based chronic disease (ABCD) is of particular interest [16] and is in line with the EASO's proposal to improve the ICD-11 diagnostic criteria for obesity based on three dimensions, namely etiology, degree of adiposity, and health risks [6]. The relevance and timeliness of these proposals is further accentuated by the current global health paradigm where the burden has moved from acute communicable diseases towards chronic noncommunicable diseases (NCDs) [17], even in low- and middle-income countries. However, momentum behind efforts to mobilize action against NCDs seems to have stalled. This requires a shift in the conceptual framework for the prevention and treatment of the ABCD of obesity, e.g., in terms of new relations between health care workers, patients, stakeholders, citizens, and society as a whole.

\section{New Advanced Diagnostic Framework}

Importantly, scientific societies both in Europe and North America recognize that obesity is a frequent, serious, complex, relapsing, and chronic disease process that represents a major public health problem. In addition, the term obesity is fraught with stigma and negativity regarding the personal character of patients with the disease [18]. A comprehensive and proactive strategy to deal with the challenges posed by the obesity epidemic in a sustainable way is needed. This requires the development and implementation of programs for early diagnosis, especially in childhood, treatment, and prevention.

The body mass index (BMI) is a simple, easy, and inexpensive way of identifying obesity [19]. However, in the elderly, muscle mass loss makes BMI a less accurate predictor, and differences in threshold indications by ethnicity have also to be specifically considered. Moreover, it has been shown that obesity defined by BMI alone is a remarkably heterogeneous condition with varying cardiovascular and metabolic manifestations across individuals, which may be different depending on gender and age $[7,20]$. Obesity complications are determined mainly by 2 pathological processes: physical forces (fat mass disease) as well as endocrine and immune responses (sick fat disease) (Fig. 1a), which are embedded in a cultural and physical context (Fig. 1b) leading to a specific ABCD stage (Fig. 1c). Hence, 
Fig. 1. Schematic diagram of the adiposity-based chronic disease (ABCD) conceptual framework of obesity, which incorporates the characteristics of adiposity including the total amount, distribution, as well as function of adipose tissue (a), the physical (human-made or built environment, i.e., food availability, urban planning, endocrine disruptors, etc.) and cultural (socio-political-economic aspects, i.e., customs, attitudes towards food and physical activity, culture, ethnicity, beliefs, policy, inequalities, and stigmatization, among others) context in which it is embedded (b), together with the clinical burden derived from the impact of the dysfunctional fat over time, which leads to the development of complications and a gender-specific disease stage (c).
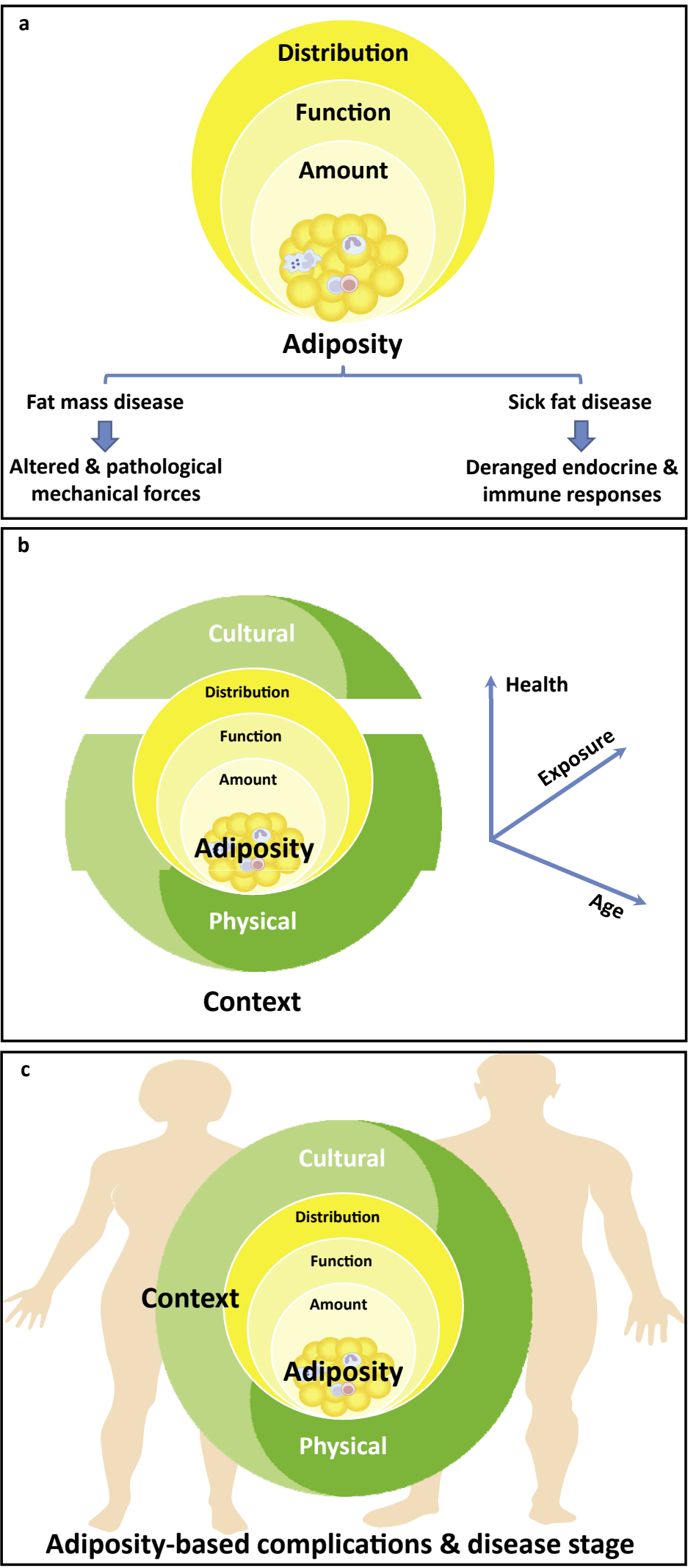
Frühbeck et al:: The ABCD of Obesity

BMI as a unique measurement of obesity does not reflect the whole complexity of the disease.

Adipose tissue is an extraordinarily dynamic, metabolically active organ involved in multiple biological processes. Adipose tissue dysfunction usually occurs due to pathological expansion of fat mass and/or unhealthy distribution of body fat, resulting in cardiometabolic complications and other related diseases [21-23]. Epidemiological, clinical, and translational studies over the past decades have provided evidence of the strong link between visceral and ectopic fat and the development of dyslipidemia, insulin resistance, hypertension, atherosclerosis, and adverse cardiac remodeling, among others [20]. Whilst being clear that visceral/ ectopic fat accumulation is a major contributor to cardiometabolic risk above and beyond the BMI $[24,25]$, implementation of fat distribution assessment into clinical practice remains a challenge. For this reason, EASO has recently argued that the simple measure BMI should continue to be used within ICD-11. However, both the degree of obesity and the associated health risk of the individual patient need to be classified additionally, in order to reach beyond the simplistic diagnosis of obesity [4]. Risk prediction, including a better evaluation of adipose tissue detrimental to health, warrants further research.

In the $\mathrm{ABCD}$ context, in which obesity is addressed as an "adiposity-based chronic disease", the incorporation of the characteristics of "adiposity" includes the total amount, distribution, as well as function of adipose tissue. In fact, body fat mass correlates with complications embodying adverse clinical endpoints that are not always adequately reflected by the BMI [16]. Body fat distribution assessment should consider the determination of the relative accumulation of visceral as well as ectopic fat depots linked to cardiometabolic disorders in comparison with the amount of subcutaneous adipose tissue, including upper- (abdominal) and lower-body (gluteofemoral) subcutaneous fat, the latter depot being associated with potential protective cardiometabolic effects when total adipose tissue mass is taken into account [20]. The functionality of adipose tissue relates to its mechanical and secretory properties, which translate into its expansion capacity as well as secretion pattern of adipokines. In fact, adipose tissue dysfunction is linked to a decreased expandability of adipocytes to accommodate the dietary energy surplus in the form of triglycerides, which seems to be related to alterations in extracellular matrix composition and fibrotic characteristics [26], as well as low-grade chronic inflammation [23]. Together, these impairments have both local and systemic effects $[26,23]$. Thus, an "adiposity-based" diagnosis allows a more specific analysis of complications determined by dysfunctional adipose tissue, based on the preponderance of scientific and clinical evidence [27].

A chronic disease is characterized by persistence of a disease state for more than 3 months, accompanied by adaptive or maladaptive processes that interact and evolve towards a steady state, which subsequently can derive in a symptom burden that can blur or mask the primary etiology. Thus, the imprecise term of obesity, which is based solely on BMI, should be replaced by a more precise diagnostic term that describes a disease state, which has the potential to promote adequate comorbidity screening and allows the application of standardized prevention and treatment protocols leading to improved patient care.

\section{Strategy and Perspectives}

The global obesity epidemic in children and adults will only worsen without thoughtful and evidence-based targeted interventions that address both the individual and society as a whole, including the environmental (socio-political-economic) context. In fact, obesity should be viewed as the main target to combat the increasing NCD epidemic. We must widen our perspective beyond the individual to create social and physical environments that promote 
health and well-being, and reduce health inequalities. The advanced diagnostic framework and chronic care model including clinical practice algorithms, together with guidelines for the comprehensive management of patients with diverse types of obesities (i.e., degree of fat mass expansion, body fat distribution) and related complications should encompass not only the biological and psychological aspects but also the socio-political-economic ones [28]. Obesity is defined as an abnormal fat accumulation that impairs health [29]. It would be mandatory to confront the causes of obesity at multiple levels to foster integrative approaches that incorporate cross-sectional collaborations between health care providers, as well as nontraditional stakeholders like welfare workers, governmental and nongovernmental organizations, the civil society, and industries. Lawyers can facilitate the translation into bold changes in social policies with a particular emphasis on decreasing health inequalities [30]. It is now time to move away from the Sisyphean task of pursuing weight loss aims alone and onto achieving other valued health goals [31]. Lifestyle intervention may reduce type 2 diabetes and cardiometabolic risk by 58\%, despite moderate weight loss [32]. Many NCDs have common etiologies, whereby a shift from a vertical to a horizontal approach will ultimately pave the path to more effective prevention and management strategies. In order to warrant adequate patient care and progress during the life course, special emphasis should be applied in teaching practicing doctors and residents, as well as other health care workers, about how to deal with the NCD epidemic, how to adequately diagnose and treat patients with obesity, and how to avoid the negative impact of body weight bias and stigmatization $[33,18]$. Moreover, pre-, postgraduate, and continuous education of health care workers should offer a much more prominent place to obesity but also lifestyle medicine into the curriculum, given its prevalence and relevance to reduce the global impact on health care systems, economies, and societies.

\section{Disclosure Statement}

L.B. has received fees for participation on the Scientific Advisory Board from Novo Nordisk. D.D. has received honoraria for lectures/counseling from MSD, Novo Nordisk, AstraZeneca, Sanofi, Pfizer, Teva, and Novartis. V.Y. has received honoraria for lectures/counseling from Novo Nordisk. E.E.B. receives grant support from the food industry such as DSM, Danone, Friesland Campina, Avebe, and Sensus, partly within the context of public-private consortia, and has received research funding from pharmaceutical companies like Novartis. She is involved in several task forces/expert groups related to the International Life Science Institute, ILSI Europe. H.T. has received honoraria for lectures from Almased, Cheplapharm, Merck, Novartis, Novo Nordisk, Roche, and Vivus.

\section{References}

1 Frühbeck G, Gómez-Ambrosi J. Control of body weight: a physiologic and transgenic perspective. Diabetologia. 2003 Feb;46(2):143-72.

2 Gluckman PD, Hanson M, Zimmet P, Forrester T. Losing the war against obesity: the need for a developmental perspective. Sci Transl Med. 2011 Jul;3(93):93cm19.

3 Villarejo C, Fernández-Aranda F, Jiménez-Murcia S, Peñas-Lledó E, Granero R, Penelo E, et al. Lifetime obesity in patients with eating disorders: increasing prevalence, clinical and personality correlates. Eur Eat Disord Rev. 2012 May;20(3):250-4.

4 NCD Risk Factor Collaboration (NCD-RisC). Trends in adult body-mass index in 200 countries from 1975 to 2014: a pooled analysis of 1698 population-based measurement studies with $19 \cdot 2$ million participants. Lancet. 2016 Apr;387(10026):1377-96.

5 Frühbeck G, Toplak H, Woodward E, Yumuk V, Maislos M, Oppert JM; Executive Committee of the European Association for the Study of Obesity. Obesity: the gateway to ill health - an EASO position statement on a rising public health, clinical and scientific challenge in Europe. Obes Facts. 2013;6(2):117-20. 
Frühbeck et al:: The ABCD of Obesity

6 Hebebrand J, Holm JC, Woodward E, Baker JL, Blaak E, Durrer Schutz D, et al. A proposal of the European Association for the Study of Obesity to improve the ICD-11 diagnostic criteria for obesity based on the three dimensions etiology, degree of adiposity and health risk. Obes Facts. 2017;10(4):284-307.

7 Blundell JE, Dulloo AG, Salvador J, Frühbeck G; EASO SAB Working Group on BMI. Beyond BMI-phenotyping the obesities. Obes Facts. 2014;7(5):322-8.

8 Frühbeck G, Toplak H, Woodward E, Halford JC, Yumuk V; European Association for the Study of Obesity. Need for a paradigm shift in adult overweight and obesity management - an EASO position statement on a pressing public health, clinical and scientific challenge in Europe. Obes Facts. 2014;7(6):408-16.

9 Yumuk V, Frühbeck G, Oppert JM, Woodward E, Toplak H. An EASO position statement on multidisciplinary obesity management in adults. Obes Facts. 2014;7(2):96-101.

10 Toplak H, Woodward E, Yumuk V, Oppert JM, Halford JC, Frühbeck G. 2014 EASO Position Statement on the use of anti-obesity drugs. Obes Facts. 2015;8(3):166-74.

11 Yumuk V, Tsigos C, Fried M, Schindler K, Busetto L, Micic D, et al.; Obesity Management Task Force of the European Association for the Study of Obesity. European guidelines for obesity management in adults. Obes Facts. 2015;8(6):402-24.

12 Frühbeck G, Sbraccia P, Nisoli E, Woodward E, Yumuk V, Farpour-Lambert NJ, et al. 2015 Milan Declaration: a call to action on obesity - an EASO position statement on the occasion of the 2015 EXPO. Obes Facts. 2016; 9(4):296-8.

13 Allison DB, Downey M, Atkinson RL, Billington CJ, Bray GA, Eckel RH, et al. Obesity as a disease: a white paper on evidence and arguments commissioned by the Council of the Obesity Society. Obesity (Silver Spring). 2008 Jun;16(6):1161-77.

14 Mechanick JI, Garber AJ, Handelsman Y, Garvey WT. American Association of Clinical Endocrinologists' position statement on obesity and obesity medicine. Endocr Pract. 2012 Sep-Oct;18(5):642-8.

15 Garvey WT, Mechanick JI, Brett EM, Garber AJ, Hurley DL, Jastreboff AM, et al.; Reviewers of the AACE/ACE Obesity Clinical Practice Guidelines. American Association of Clinical Endocrinologists and American College of Endocrinology Comprehensive Clinical Practice Guidelines for Medical Care of Patients with Obesity. Endocr Pract. 2016 Jul;22 Suppl 3:1-203.

16 Mechanick JI, Hurley DL, Garvey WT. Adiposity-based chronic disease as a new diagnostic term: The American Association of Clinical Endocrinologists and American College of Endocrinology Position Statement. Endocr Pract. 2017 Mar;23(3):372-8.

17 Horton R, Sargent J. 2018 must be the year for action against NCDs. Lancet. 2018 May;391(10134):1971-3.

18 Ramos Salas X, Alberga AS, Cameron E, Estey L, Forhan M, Kirk SF, et al. Addressing weight bias and discrimination: moving beyond raising awareness to creating change. Obes Rev. 2017 Nov;18(11):1323-35.

19 Adab P, Pallan M, Whincup PH. Is BMI the best measure of obesity? BMJ. 2018 Mar;360:k1274.

20 Neeland IJ, Poirier P, Després JP. Cardiovascular and metabolic heterogeneity of obesity: clinical challenges and implications for management. Circulation. 2018 Mar;137(13):1391-406.

21 Gómez-Ambrosi J, Salvador J, Páramo JA, Orbe J, de Irala J, Diez-Caballero A, et al. Involvement of leptin in the association between percentage of body fat and cardiovascular risk factors. Clin Biochem. 2002 Jun; 35(4): 315-20.

22 Rodríguez A, Ezquerro S, Méndez-Giménez L, Becerril S, Frühbeck G. Revisiting the adipocyte: a model for integration of cytokine signaling in the regulation of energy metabolism. Am J Physiol Endocrinol Metab. 2015 Oct;309(8):E691-714.

23 Goossens GH. The metabolic phenotype in obesity: fat mass, body fat distribution, and adipose tissue function. Obes Facts. 2017;10(3):207-15.

24 Frühbeck G, Gómez-Ambrosi J. Rationale for the existence of additional adipostatic hormones. FASEB J. 2001 Sep;15(11):1996-2006.

25 Trim W, Turner JE, Thompson D. Parallels in immunometabolic adipose tissue dysfunction with ageing and obesity. Front Immunol. 2018 Feb;9:169.

26 Sun K, Tordjman J, Clément K, Scherer PE. Fibrosis and adipose tissue dysfunction. Cell Metab. 2013 Oct;18(4): 470-7.

27 Gadde KM, Martin CK, Berthoud HR, Heymsfield SB. Obesity: pathophysiology and Management. J Am Coll Cardiol. 2018 Jan;71(1):69-84.

28 Frühbeck G, Kiortsis DN, Catalán V. Precision medicine: diagnosis and management of obesity. Lancet Diabetes Endocrinol. 2018 Mar;6(3):164-6.

29 World Health Organization. Obesity. Preventing and managing the global epidemic. Report of a WHO consultation on obesity. WHO/NUT/NCD/981. Geneva: WHO; 1998.

30 Cheng JK. Confronting the social determinants of health-obesity, neglect, and inequity. N Engl J Med. 2012 Nov;367(21):1976-7.

31 Frühbeck G. Bariatric and metabolic surgery: a shift in eligibility and success criteria. Nat Rev Endocrinol. 2015 Aug;11(8):465-77.

32 Penn L, White M, Lindström J, den Boer AT, Blaak E, Eriksson JG, et al. Importance of weight loss maintenance and risk prediction in the prevention of type 2 diabetes: analysis of European Diabetes Prevention Study RCT. PLoS One. 2013;8(2):e57143.

33 Silk AW, McTigue KM. Reexamining the physical examination for obese patients. JAMA. 2011 Jan;305(2): 193-4. 